\title{
KIDA'S THEOREM FOR A CLASS OF NONNORMAL EXTENSIONS
}

\author{
ROBERT GOLD AND MANOHAR MADAN
}

(Communicated by Larry J. Goldstein)

\begin{abstract}
Let $E, F$ be $\mathbf{Z}_{p}$-fields of $C M$-type such that $E / F$ is an extension of degree $p$. Let $L$, the normal closure of $E / F$, be such that $\mathrm{Gal}(L / F)$ has a normal subgroup of order $p$. Denote the fixed field of this group by $K$. We prove a Kida type formula which describes the minus part of the Iwasawa lambda invariant of $E$ in terms of the lambda invariants of $F$ and $K$.
\end{abstract}

1. Introduction. Let $p$ be an odd prime. Let $\mathbf{Q}_{n}$ be the unique cyclic extension of degree $p^{n}$ contained in the cyclotomic field of $p^{n+1}$ th roots of unity and $\mathbf{Q}_{\infty}=$

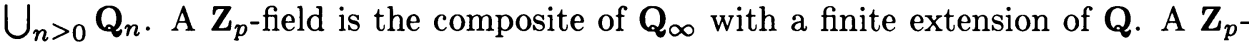
field $F$ of $C M$-type is a totally imaginary $\mathbf{Z}_{p}$-field which is a quadratic extension of a totally real $\mathbf{Z}_{p}$-field $F^{+}$. Let $A_{F}^{-}$denote the subgroup of the $p$-class group of $F$ consisting of classes $c$ such that $c^{J}=c^{-1}, J$ denoting complex conjugation.

A well-known conjecture of Iwasawa on the vanishing of the $\mu$-invariant implies that $A_{F}^{-} \cong\left(\mathbf{Q}_{p} / \mathbf{Z}_{p}\right)^{\lambda_{F}^{-}}$for some nonnegative integer $\lambda_{F}^{-}$, where $\mathbf{Q}_{p}, \mathbf{Z}_{p}$ denote the field of $p$-adic numbers and the ring of $p$-adic integers, respectively. Our object, in this paper, is to prove the following generalization of Kida's Theorem [K].

THEOREM. Let $E, F$ be $\mathbf{Z}_{p}$-fields of $C M$-type such that $E / F$ is an extension of degree $p$. Let $L$, the normal closure of $E / F$, be such that $\operatorname{Gal}(L / F)$ has a normal subgroup of order $p$. Denote the fixed field of this group by K. Then $\mu_{K}^{-}=0$ implies $\mu_{F}^{-}=\mu_{E}^{-}=0$, and

$$
\lambda_{E}^{-}=\lambda_{F}^{-}+\frac{p-1}{[K: F]}\left(\lambda_{K}^{-}+t-\delta\right)
$$

where $t$ is the number of non-p-primes of $K^{+}$that ramify in $L^{+}$and split in $K$, and $\delta$ is 1 or 0 according as $K$ does or does not contain the pth roots of unity.

We give two proofs of this theorem, one arithmetic-algebraic and the other analytic. The first proof is based on an analysis of the action of $\operatorname{Gal}(L / F)$ on the $p$-elementary subgroup of $A_{L}^{-}$. It uses some facts proved in [GM]. The analytic proof uses relations between nonabelian $p$-adic $L$-functions. As in $[\mathbf{S}]$, the critical fact used in this proof is a relation, due to Iwasawa, between $p$-adic $L$-functions and Iwasawa invariants.

Kida's theorem is the analogue of a formula of Deuring and Safarevic, a special case of which relates the Hasse-Witt invariants of the function fields of a cyclic extension of degree $p$ such that the field of constants $k$ is an algebraically closed

Received by the editors January 7, 1987 and, in revised form, August 13, 1987.

1980 Mathematics Subject Classification (1985 Revision). Primary 11R23; Secondary 11R29. 
field of characteristic $p$. In this case, Rück [R] has proved the corresponding generalization. His proof is analytic. Our arithmetic-algebraic proof remains valid in the function field case and, in fact, it holds also in the truly analogous situation when $k$ is the $\mathbf{Z}_{p}$-extension of a finite field. Using some facts from [DM], the reader can easily supply the details.

2. The arithmetic algebraic proof. By a theorem of Iwasawa $[\mathbf{I}], \mu_{K}^{-}=0$ implies $\mu_{L}^{-}=0$. Thus, the $p$-elementary subgroup of $A_{L}^{-}$is finite of rank $\lambda_{L}^{-}$. Since the kernels of the conorm maps $A_{F}^{-} \rightarrow A_{E}^{-}, A_{E}^{-} \rightarrow A_{L}^{-}$are finite, it follows that the $p$-elementary subgroups of $A_{E}^{-}, A_{F}^{-}$are also finite, i.e. $\mu_{F}^{-}=0, \mu_{E}^{-}=0$.

The assumption that $\operatorname{Gal}(L / K)$ is a normal subgroup of order $p$ of the Galois group of the normal closure $L / F$ of $E / F$ implies that $\mathrm{Gal}(L / F)$ is a semidirect product of $\mathrm{Gal}(L / K)$ and $\mathrm{Gal}(L / E)$, the latter is a cyclic group of order $d$ dividing $p-1$. Let $G=\operatorname{Gal}(L / K)=\langle\sigma\rangle, \operatorname{Gal}(L / E)=\langle\tau\rangle$ and $\tau \circ \tau^{-1}=\sigma^{r}$. Let $X_{L}$ denote the $p$-elementary subgroup of $A_{L}^{-}$. For $i=1,2, \ldots, p-1, p$, let

$$
X_{i}=\left\{c: c \in X_{L}, c^{(1-\sigma)^{i}}=1\right\} .
$$

We have the descending chain of $\tau$-invariant subspaces

$$
X_{L}=X_{p} \supset X_{p-1} \supset \cdots \supset X_{2} \supset X_{1} \supset X_{0}=(1) .
$$

Denoting by $\mathbf{F}_{p}$ the finite field with $p$ elements, we recall the following facts from [GM].

$$
\begin{gathered}
\operatorname{dim}_{\mathbf{F}_{p}} X_{1}= \begin{cases}\lambda_{K}^{-}+t-\delta, & \text { if } t>0, \\
\lambda_{K}^{-}, & \text {if } t=0,\end{cases} \\
\operatorname{dim}_{\mathbf{F}_{p}}\left(X_{p} / X_{p-1}\right)= \begin{cases}\lambda_{K}^{-}, & \text {if } t>0, \\
\lambda_{K}^{-}-\delta, & \text { if } t=0\end{cases}
\end{gathered}
$$

For the divisible module $A_{L}^{-}$, we have

$$
A_{L}^{-} \cong A_{1}^{a_{1}} \oplus A_{p-1}^{a_{p-1}} \oplus A_{p}^{a_{p}}
$$

where $A_{1}$ denotes the trivial $G$-module $\mathbf{Q}_{p} / \mathbf{Z}_{p}, A_{p}$ denotes the divisible regular representation $\left(\mathbf{Q}_{p} / \mathbf{Z}_{p}\right)[x] / x^{p}-1$, and $A_{p-1}$ denotes the divisible faithful representation $\left(\mathbf{Q}_{p} / \mathbf{Z}_{p}\right)[x] / x^{p-1}+\cdots+x+1$, for uniquely determined integers $a_{1}, a_{p-1}$, $a_{p}$.

We separate the ramified and the unramified cases.

$L / K$ ramified. As shown in $[\mathbf{G M}]$, in this case, $H^{-1}\left(G, A_{L}^{-}\right)=1, H^{-1}\left(G, A_{1}\right) \cong$ $\mathbf{Z} / p \mathbf{Z}$. Therefore, restricting the decomposition (3) to $X_{L}$, we have

$$
X_{L} \cong\left(\frac{\mathbf{F}_{p}[x]}{(1-x)^{p-1}}\right)^{a_{p-1}} \oplus\left(\frac{\mathbf{F}_{p}[x]}{(1-x)^{p}}\right)^{a_{p}}
$$

Using (2), it follows that

$$
\begin{gathered}
\operatorname{dim}_{\mathbf{F}_{p}}\left(X_{i} / X_{i-1}\right)=a_{p-1}+a_{p}=\lambda_{K}^{-}+t-\delta, \quad i=1,2, \ldots, p \\
\operatorname{dim}_{\mathbf{F}_{p}}\left(X_{p} / X_{p-1}\right)=a_{p}=\lambda_{K}^{-} .
\end{gathered}
$$


To evaluate the order of $X_{E}$, the $p$-elementary subgroup of $A_{E}^{-}$, we observe that $(d, p)=1$ implies that it is injected in $X_{L}$ and can be identified with the subgroup $X_{L}^{\langle\tau\rangle}$ of $X_{L}$ consisting of classes which are invariant under $\tau$. We consider the map

$$
X_{i} / X_{i-1} \rightarrow X_{1}, \quad i=1,2, \ldots, p-1,
$$

defined by

$$
\bar{x}=x X_{i-1} \rightarrow x^{(1-\sigma)^{i-1}}=y .
$$

By (2), this is an isomorphism of groups. Moreover

$$
\begin{aligned}
\bar{x}^{\tau}=\bar{x}=\overline{x^{\tau}} & \Leftrightarrow\left(x^{\tau}\right)^{(1-\sigma)^{i-1}}=x^{(1-\sigma)^{i-1}} \\
& \Leftrightarrow x^{\tau(1-\sigma)^{i-1} \tau^{-1}}=\left(x^{(1-\sigma)^{i-1}}\right)^{\tau^{-1}} \\
& \Leftrightarrow x^{\left(1-\sigma^{r}\right)^{i-1}}=\left(x^{(1-\sigma)^{i-1}}\right)^{\tau^{-1}} \\
& \Leftrightarrow\left(x^{(1-\sigma)^{i-1}}\right)^{r^{i-1}}=\left(x^{(1-\sigma)^{i-1}}\right)^{\tau-1} \\
& \Leftrightarrow y^{r^{i-1}}=y^{\tau^{-1}} \\
& \Leftrightarrow y^{\tau}=y^{r^{1-i}} .
\end{aligned}
$$

Thus, the $\tau$-invariant elements correspond to the eigenspace of $X_{1}$ for the eigenvalue $r^{1-i}$. Also, $(1-\sigma)^{p-1}$ maps $X_{p} / X_{p-1}$ onto $X_{K}$ injected in $X_{L}$ and the $\tau$-invariant elements of $A_{K}^{-}$are precisely the elements of $A_{F}^{-}$. Considering that $d$ is the order of $r$ modulo $p$ and $\operatorname{dim}_{\mathbf{F}_{p}} X_{1}=t+\lambda_{K}^{-}-\delta$, we have, in this ramified case,

$$
\lambda_{E}^{-}=\lambda_{F}^{-}+\frac{p-1}{d} \operatorname{dim}_{F_{p}} X_{1}=\lambda_{F}^{-}+\frac{p-1}{d}\left(\lambda_{K}^{-}+\tau-\delta\right) .
$$

$L / K$ unramified. As shown in $[\mathbf{G M}]$, in this case $H^{0}\left(G, A_{L}^{-}\right)=1, H^{0}\left(G, A_{p-1}\right)=$ 1. Therefore, $a_{p-1}=0$ in the decomposition (3). Thus, restricting to $X_{L}$, we have

$$
X_{L} \cong\left(\frac{\mathbf{F}_{p}[x]}{1-x}\right)^{a_{1}} \oplus\left(\frac{\left.\mathbf{F}_{[} x\right]}{(1-x)^{p}}\right)^{a_{p}}
$$

Using (2), it follows that

$$
\begin{gathered}
\operatorname{dim}_{\mathbf{F}_{p}}\left(X_{i} / X_{i-1}\right)=a_{p}=\lambda_{K}^{-}-\delta, \quad i=2, \ldots, p . \\
\operatorname{dim}_{\mathbf{F}_{p}} X_{1}=a_{1}+a_{p}=\lambda_{K}^{-} .
\end{gathered}
$$

We consider the isomorphisms

$$
X_{i} / X_{i-1} \rightarrow X_{2}^{1-\delta}, \quad i=2,3, \ldots, p
$$

induced by

$$
\bar{x}=x X_{i-1} \rightarrow x^{(1-\sigma)^{i-1}} .
$$

As in the ramified case, one can show that the $\tau$-invariant elements correspond to the eigenspace for the eigenvalue $r^{1-i}$. Further the space of $\tau$-invariant elements of $X_{1}$ has dimension $\lambda_{F}^{-}$. Therefore, $\lambda_{E}^{-}$, the dimension of the $\tau$-invariant elements of $X_{p}$ is given by

$$
\lambda_{E}^{-}=\lambda_{F}^{-}+\frac{p-1}{d}\left(\lambda_{K}^{-}-\delta\right) .
$$

This completes the arithmetic-algebraic proof of the theorem. 
3. The analytic proof. This proof will involve a combination of the techniques of Rück [R] and Sinnott $[\mathbf{S}]$.

First we descend to the finite level. There exist finite number fields $F_{0}, E_{0}, K_{0}$, $L_{0}$ such that $L=L_{0} \cdot \mathbf{Q}_{\infty}, F=F_{0} \cdot \mathbf{Q}_{\infty}, \operatorname{Gal}(L / F) \cong \operatorname{Gal}\left(L_{0} / F_{0}\right)$, etc.

Let $\chi_{K}$ denote the regular character of $\operatorname{Gal}\left(L_{0} / K_{0}\right)$ minus the trivial character of that group. Let $\chi_{E}$ be defined similarly for $\operatorname{Gal}\left(L_{0} / E_{0}\right)$ and $\chi_{F}$ for $\operatorname{Gal}\left(K_{0} / F_{0}\right)$. Let $\widehat{\chi}_{E}, \widehat{\chi}_{K}$ be the characters of $\operatorname{Gal}\left(L_{0} / F_{0}\right)$ induced from $\chi_{E}, \chi_{K}$ and $\bar{\chi}_{F}$ the character of $\operatorname{Gal}\left(L_{0} / F_{0}\right)$ deduced from $\chi_{F}$ in the obvious way. Then Rück [R] proves

$$
d \cdot \widehat{\chi}_{E}=d \cdot \bar{\chi}_{F}+(d-1) \widehat{\chi}_{K} .
$$

We will use Sinnott's method (and notation) to deduce from (6) a relation among $p$-adic $L$-functions and, consequently, a relation on $\lambda$-invariants. Let $S$ be the set of places (in any appropriate field) which ramify in $L_{0} / F_{0}^{+}$together with all places over $p$. Now (6) gives the following relation among complex $L$-functions with Euler factors at $S$ omitted.

$$
\begin{aligned}
& \prod_{\substack{\psi \in \operatorname{Gal}\left(L_{0} / E_{0}\right)^{\wedge} \\
\psi \neq 1}} L_{S}\left(s, \rho \psi, E_{0}^{+}\right)^{d}=\prod_{\substack{\psi \in \operatorname{Gal}\left(K_{0} / F_{0}\right)^{\wedge} \\
\psi \neq 1}} L_{S}\left(s, \rho \psi, F_{0}^{+}\right) \\
& \prod_{\substack{\psi \in \operatorname{Gal}\left(L_{0} / K_{0}\right)^{\wedge} \\
\psi \neq 1}} L_{S}\left(s, \rho \psi, K_{0}^{+}\right)^{d-1}
\end{aligned}
$$

where $\rho=\varepsilon \theta$ for $\varepsilon$ the odd quadratic character of $F_{0} / F_{0}^{+}, L_{0} / L_{0}^{+}$, etc. and $\theta$ the Teichmüller character of $F_{0}^{+}\left(\zeta_{p}\right) / F_{0}^{+}$.

Using the standard properties of complex $L$-functions we can rewrite this equation as

$$
\frac{L_{S}\left(s, \rho, L_{0}^{+}\right)^{d}}{L_{S}\left(s, \rho, E_{0}^{+}\right)^{d}}=\frac{L_{S}\left(s, \rho, K_{0}^{+}\right)^{d}}{L_{S}\left(s, \rho, F_{0}^{+}\right)^{d}} \cdot \frac{L_{S}\left(s, \rho, L_{0}^{+}\right)^{d-1}}{L_{S}\left(s, \rho, K_{0}^{+}\right)^{d-1}}
$$

which simplifies to

$$
\frac{L_{S}\left(s, \rho, L_{0}^{+}\right)}{L_{S}\left(s, \rho, K_{0}^{+}\right)} \cdot L_{S}\left(s, \rho, F_{0}^{+}\right)^{d}=L_{S}\left(s, \rho, E_{0}^{+}\right)^{d} .
$$

This yields

$$
\left\{\prod_{\substack{\psi \in \operatorname{Gal}\left(L_{0} / K_{0}\right)^{\wedge} \\ \psi \neq 1}} L_{S}\left(s, \rho \psi, K_{0}^{+}\right)\right\} \cdot L_{S}\left(s, \rho, F_{0}^{+}\right)^{d}=L_{S}\left(s, \rho, E_{0}^{+}\right)^{d} .
$$

Let $\widetilde{L}_{S}(\chi, k, T)$ be the Iwasawa power series defined by the interpolation property

$$
\widetilde{L}_{S}\left(\chi, k, \kappa^{1-n}-1\right)=L_{S}\left(1-n, \chi \theta^{-n}, k\right)
$$

where $\kappa$ generates $\operatorname{Aut}_{k\left(\zeta_{p}\right)}\left(k\left(\zeta_{p} \infty\right)\right)$ viewed as a subgroup of $1+p \mathbf{Z}_{p}$.

Our basic equation holds for $L_{S}(s, \chi, k)$ replaced by $\widetilde{L}_{S}(\chi, k, T)$. 
Denoting (as in [S]) the $\lambda$-invariant of the power series $\tilde{L}_{S}(\chi, k, T)$ by $\lambda_{S}(\chi, k)$, we obtain

$$
\sum_{\substack{\psi \in \operatorname{Gal}\left(L_{0} / K_{0}\right)^{\gamma} \\ \psi \neq 1}} \lambda_{S}\left(\rho \psi, K_{0}^{+}\right)+d \cdot \lambda_{S}\left(\rho, F_{0}^{+}\right)=d \cdot \lambda_{S}\left(\rho, E_{0}^{+}\right) .
$$
have

By Proposition 2.1 in Sinnott $[\mathbf{S}]$ and the fact that $\operatorname{Gal}\left(L_{0} / K_{0}\right)$ has order $p$, we

$$
\frac{p-1}{d} \lambda_{S}\left(\rho, K_{0}^{+}\right)+\lambda_{S}\left(\rho, F_{0}^{+}\right)=\lambda_{S}\left(\rho, E_{0}^{+}\right) .
$$

It remains now to relate each $\lambda_{S}\left(\rho, k_{0}^{+}\right)$to the corresponding $\lambda_{k}^{-}$. But $\lambda_{k}^{-}=$ $\lambda_{S^{\prime}}\left(\rho, k_{0}^{+}\right)+\delta(k)$ [S, Proposition 3.1] where $S^{\prime}$ is the set of places of $k_{0}^{+}$which ramify in $k_{0} \cdot Q_{\infty} / k_{0}^{+}$. The required relation is given [S, Lemma 2.1$]$ by

$$
\lambda_{S}\left(\rho, k_{0}^{+}\right)=\lambda_{k}^{-}+\sum_{\mathscr{P}}^{(k)} g(\mathscr{P})-\delta(k) \quad \text { for } k=F, E, K
$$

where the sum is over all places $\mathscr{P}$ in $S / k$ which are not ramified in $k / k_{0}^{+}$such that $\mathscr{P}$ is split in $k_{0} / k_{0}^{+}, g(\mathscr{P})$ is the number of places of $k^{+}$lying over $\mathscr{P}$, and $\delta(k)=1,0$ as $k$ contains a $p$ th-root of unity or not.

In light of (7) and (8), the theorem will follow if we can show that

$$
\frac{p-1}{d} \sum_{\mathscr{P}}^{(K)} g(\mathscr{P})+\sum_{\mathscr{P}}^{(F)} g(\mathscr{P})-\sum_{\mathscr{P}}^{(E)} g(\mathscr{P})=\frac{p-1}{d} t .
$$

This equality can be verified by considering the contribution to both members of each non- $p$-prime $\mathscr{P}$ of $F_{0}^{+}$which is ramified in $L_{0} / F_{0}^{+}$. This is achieved by a nontrivial but routine and somewhat tedious examination of cases depending on the splitting behavioir of $\mathscr{P}$ in $L_{0} / F_{0}^{+}$. We omit the proof.

4. Remarks. 1. As stated in the Introduction, Kida's Theorem is an analogue of a theorem of Deuring and Safarevic. It may also be viewed as a formal analogue of the Riemann-Hurwitz genus formula.

2. Our theorem generalizes Kida's Theorem to a class of extensions of degree $p$. The restriction to extensions of degree $p$ is not essential. Using induction, it can be routinely extended (as in $[\mathbf{K}, \mathbf{S a}, \mathbf{S}]$ ) to extensions $E / F$ such that for the normal closure $L, \operatorname{Gal}(L / F)$ is the semidirect product of the normal subgroup $\operatorname{Gal}(L / K)$ of $p$-power order and the cyclic subgroup $\operatorname{Gal}(L / E)$ of order dividing $p-1$.

3 . We give an example of the application of our theorem.

Let $F=\mathbf{Q}(\sqrt{-6})$ and $E=\mathbf{Q}(\sqrt{-6}, \alpha)$ where $\alpha$ is a root of $x^{3}-11 x-11$. The field $\mathbf{Q}(\alpha)$ is a totally real cubic of discriminant $11^{2} \cdot 17$ and normal closure $\mathbf{Q}(\alpha, \sqrt{17})$. Therefore $K^{+}=\mathbf{Q}(\sqrt{17}), K=\mathbf{Q}(\sqrt{17}, \sqrt{-6}), L=\mathbf{Q}(\sqrt{17}, \sqrt{-6}, \alpha)$ and 11 is totally ramified in $L / K$. Since 3 does not divide the class number of $K$ and there is a unique prime over 3 in $K$, the invariant $\lambda_{K}^{-}, \lambda_{F}^{-}$are both zero [W]. It is easy to check that $t=1$ and $\delta=0$. Hence by the formula of our theorem, $\lambda_{E}^{-}=1$ and $\lambda_{L}^{-}=2$. 


\section{REFERENCES}

[DM] J. D'Mello and M. Madan, Class group rank relations in $\mathbf{Z}_{l}$-extensions, Manuscripta Math. 41 (1983), 75-107.

[GM] R. Gold and M. Madan, Galois representations of Iwasawa modules, Acta Arith. 46 (1986), 243-255.

[I] K. Iwasawa, On the $\mu$-invariants of $\mathbf{Z}_{l}$-extensions, Number Theory, Algebraic Geometry and Commutative Algebra, Kinokuniya, Tokyo, 1973.

[K] Y. Kida, $l$-extensions of $C M$-fields and cyclotomic invariants, J. Number Theory 12 (1980), 519-528.

[R] H.-G. Rück, Hass-Witt-invariants and dihedral extensions, Math. Z. 191 (1986), 513-517.

[Sa] I. R. Safarevic, On p-extensions, Amer. Math. Soc. Transl. (2) 4 (1954).

[S] W. Sinnott, On p-adic L-functions and the Riemann-Hurwitz genus formula, Compositio Math. 53 (1984), 3-17.

[W] L. Washington, Introduction to cyclotomic fields, Springer-Verlag, Berlin and New York, 1982.

Department of Mathematics, Ohio State University, Columbus, Ohio 43210 